This item was submitted to Loughborough's Institutional Repository (https://dspace.lboro.ac.uk/) by the author and is made available under the following Creative Commons Licence conditions.

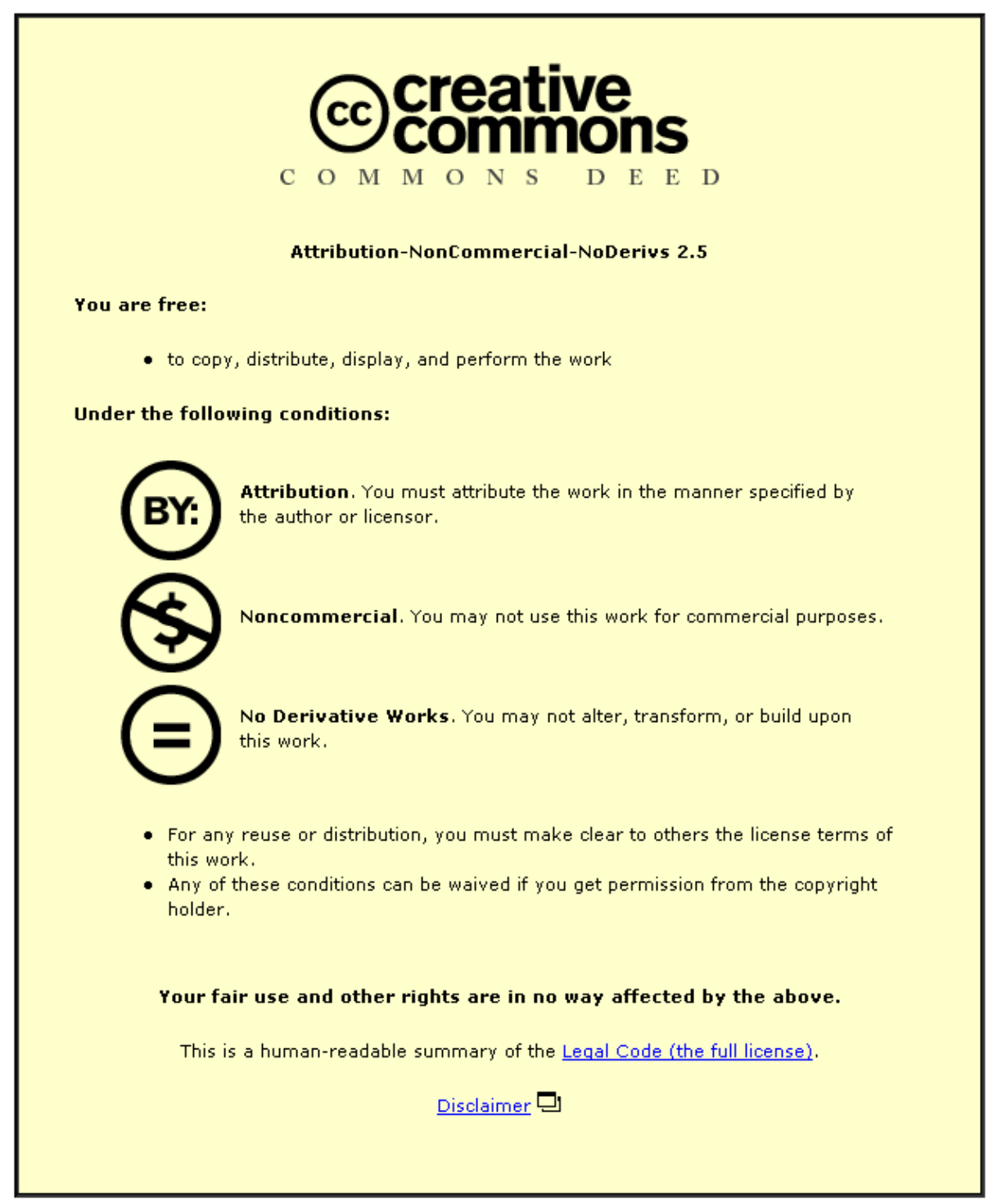

For the full text of this licence, please go to: http://creativecommons.org/licenses/by-nc-nd/2.5/ 


\title{
Sheath Dynamics in Radio-Frequency Atmospheric Glow Discharges
}

\author{
J. J. Shi and M. G. Kong
}

\begin{abstract}
This paper presents images of dynamic sheath evolution in a radio-frequency atmospheric pressure glow discharge at 13.56 MHz. Electron mean energy, electron production rate, electron density, and electric field are used to highlight volumetric gas ionization at low-current densities and localized gas ionization at large current densities.
\end{abstract}

Index Terms-Atmospheric pressure glow discharges, glow modes, plasma dynamics, sheath, simulation.

A TMOSPHERIC pressure glow discharges (APGDs) are currently a subject of active research, partly due to their immense potentials for wide-ranging application and partly due to fresh scientific challenges presented by these newly identified gas plasmas [1]. At radio-frequencies in the mega-hertz range, APGD are typically generated between two metallic electrodes without dielectric barrier. These high-frequency capacitive glow plasmas have been studied for their current-voltage characteristics, optical emission and reaction chemistry [2]-[4]. While these studies are important in capturing the macroscopic features of radio frequency (RF) APGD, the corresponding underpinning physiochemical processes on the microscopic scale remain relatively unknown. A sound understanding of such underpinning physiochemical processes is highly desirable, as it offers valuable guidance to effective control of plasma stability and chemical reactivity. Recognizing that many underpinning processes are determined by behaviors of the sheath region near the electrodes, we present in this paper images of dynamic evolution of APGD sheath as a contribution to a full understanding of APGD.

Our approach is computational as this allows for a more effective access to plasma dynamic information than diagnostics methodologies that are at present relatively under-developed for APGD. Our plasma model is based on a self-consistent and continuum treatment developed for RF APGD, and its predictions of the current and voltage characteristics agree favorably with relevant experimental data [2] and numerical results [5]. We consider a $13.56-\mathrm{MHz}$ glow discharge generated in an atmospheric helium gap of $2.5 \mathrm{~mm}$ and at gas pressure of 600 torr. While impurity gases, such as nitrogen, are known to be important in APGD, we decide not to include their effect for this study, partly because the general trend of sheath dynamics in RF APGD is found numerically to be independent of the inclusion of impurity nitrogen and partly because it is useful to distinguish

Manuscript received June 21, 2004; revised September 8, 2004.

The authors are with the Department of Electronic and Electrical Engineering, Loughborough University, Leicestershire LE11 3TU, U.K. (e-mail: m.g.kong@lboro.ac.uk).

Digital Object Identifier 10.1109/TPS.2005.844999 the generic characteristic of sheath dynamics from nitrogen-dependent effects. Consequently, our plasma model considers six species, namely electrons $e$, helium ions $\mathrm{He}^{+}$and $\mathrm{He}_{2}^{+}$, excited helium $\mathrm{He}^{*}$, excited helium dimer $\mathrm{He}_{2}^{*}$, and background helium atoms. Eleven chemical reactions are considered including direct ionization, excitation, deexcitation, charge transfer from atomic helium ions to dimer helium ions, step-wise ionizations, and recombination [5].

Fig. 1 shows time-averaged spatial profiles of (a) the electron mean energy and (b) the electron generation rate over a wide current density range. The two large humps near the two electrodes at $x=0 \mathrm{~cm}$ and $x=0.25 \mathrm{~cm}$, respectively, are the two sheath regions. In general, these are regions of the largest electric field, the greatest electron mean energy and the most efficient electron production. It is worth emphasizing that the absolute values of the electron mean energy and the electron production rate are much higher at large current densities.

The spatial profile of the normalized electron mean energy follows a general pattern of rising rapidly from the electrode surface to reach its maximum and then undergoing a gradual decaying toward the center of the plasma bulk. However, at low-current densities, both the rising phase and the decaying phase are more gradual. At $7 \mathrm{~mA} / \mathrm{cm}^{2}$, the delaying phase reduces the electron mean energy to a minimum value of about $70 \%$ of its peak value at the center of the gap. In contrast at $80 \mathrm{~mA} / \mathrm{cm}^{2}$, the minimum electron mean energy is less than $5 \%$ of its peak value at $300 \mu \mathrm{m}$ from the electrode. It is interesting to note that above $60 \mathrm{~mA} / \mathrm{cm}^{2}$, the minimum electron mean energy is not at the gap center but near the sheath-bulk boundary. In addition the maximum electron mean energy is reached closer to the electrodes at higher current densities. At $80 \mathrm{~mA} / \mathrm{cm}^{2}$, this is reached at $71 \mu \mathrm{m}$ whereas it is at $360 \mu \mathrm{m}$ with the current density reduced to $7 \mathrm{~mA} / \mathrm{cm}^{2}$. Similar trends are observed in the spatial profile of the electron production rate. These observations show that the RF APGD exhibits a volumetric nature at low-current densities. As the current density increases, electron production and hence other key physiochemical processes become localized in the boundary region between the sheath and the plasma bulk. This evolution dependence on current density is similar to that observed in low-pressure capacitively-coupled glow discharges [6] and in atmospheric pressure direct current glow discharges [7].

In summary, a computational study of radio-frequency atmospheric helium glow discharges has been used to unravel their sheath dynamics in terms of their electron mean energy and their electron production rate. Plasma images captured by these key quantities highlight volumetric gas discharge at low-current densities and localized gas discharge at large current densities. 

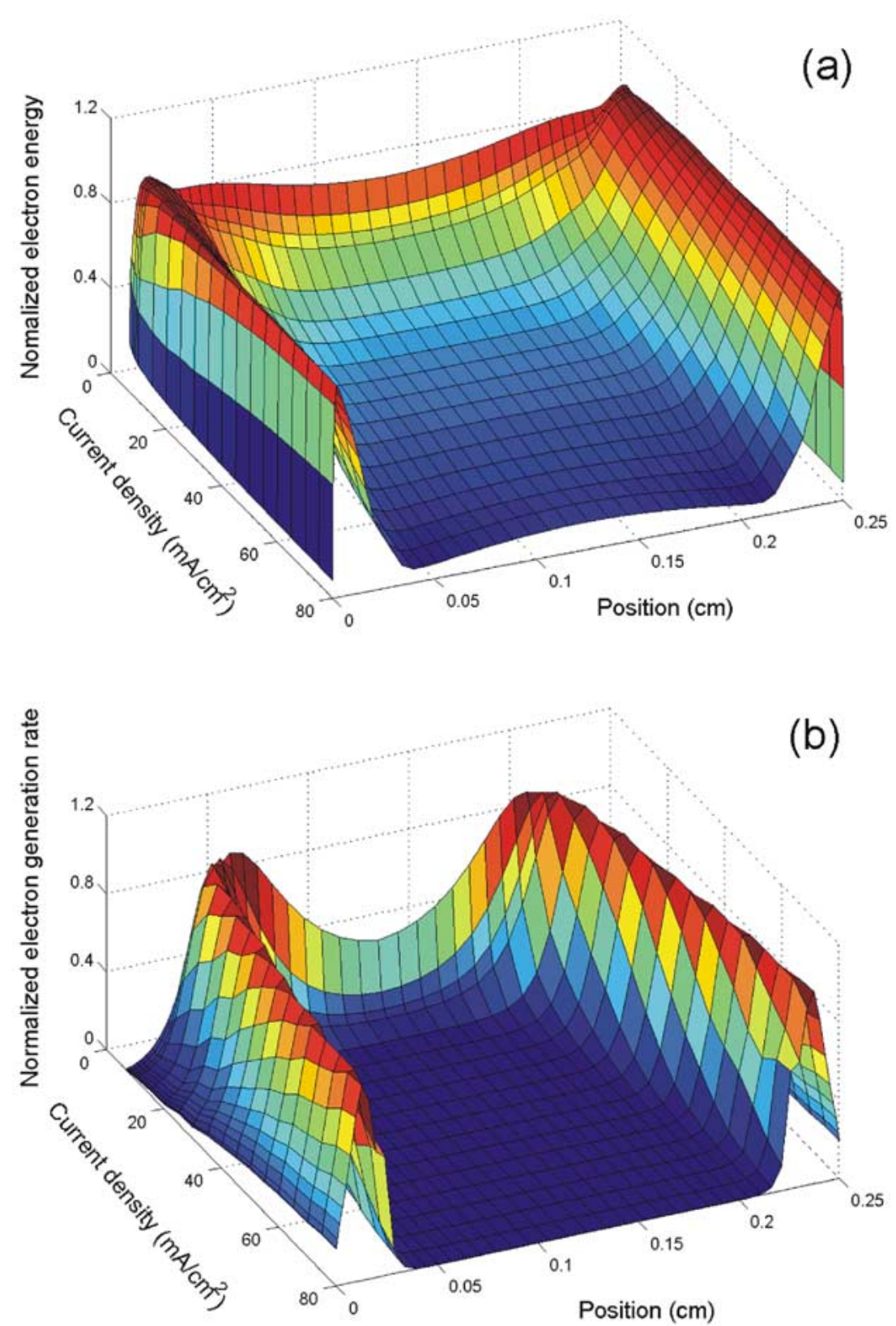

Fig. 1. Time-averaged spatial profile of normalized electron mean energy over a wide current density range from 7 to $80 \mathrm{~mA} / \mathrm{cm}^{2}$ for a helium RF APGD.

For the conditions used for our examples, the boundary between the two different regimes is approximately $12 \mathrm{~mA} / \mathrm{cm}^{2}$.

\section{REFERENCES}

[1] E. E. Kunhardt, "Generation of large-volume, atmospheric-pressure nonequilibrium plasmas," IEEE Trans. Plasma Sci., vol. 28, no. 1, pp. 189-200, Feb. 2000.

[2] J. Park, I. Henins, H. W. Herrmann, G. S. Selwyn, and R. F. Hicks, "Discharge phenomena of an atmospheric pressure radio-frequency capacitive plasma source," J. Appl. Phys., vol. 89, pp. 20-28, Jan. 2001.
[3] J. J. Shi, X. T. Deng, R. Hall, J. D. Punnett, and M. G. Kong, "Three modes in a radio frequency atmospheric pressure glow discharge," $J$. Appl. Phys., vol. 94, pp. 6303-6310, Nov. 2003.

[4] J. Y. Jeong, J. Park, I. Hennis, S. E. Babayan, V. J. Yu, G. S. Selwyn, G. Ding, and R. F. Hicks, "Reaction chemistry in the afterglow of an oxygen-helium, atmospheric-pressure plasma," J. Phys. Chem. A., vol. 104, pp. 8027-8032, Aug. 2000.

[5] X. Yuan and L. L. Raja, "Role of trace impurities in large-volume noble gas atmospheric-pressure glow discharges," Appl. Phys. Lett., vol. 81, pp. 814-816, Jul. 2002.

[6] Yu. P. Raizer, M. N. Shneider, and N. A. Yatsenko, Radio-Frequency Capacitive Discharges, FL: CRC, 1995.

[7] J. Shi and M. G. Kong, "Cathode fall characteristics in a dc atmospheric pressure glow discharge," J. Appl. Phys., vol. 94, pp. 5504-5513, Nov. 2003. 